\title{
Interactive comment on "Leaf waxes in litter and topsoils along a European transect" by I. Schäfer et al.
}

\section{Anonymous Referee \#2}

Received and published: 10 August 2016

\section{General comments}

The manuscript "Leaf waxes in litter and topsoils along a European transect" by Imke Schäfer and colleagues studies n-alkanes and n-alkanoic acids in litter and topsoils of grasslands, deciduous and coniferous forests along a transect across Europe.

In general, the manuscript is already in a very good shape and pleasant to read through. The presentation of data in figures (and supplementary tables) is of high quality and the conclusions that the authors draw are supported by the results. The study nicely shows how distribution patterns and several proxies and ratios of plant wax compounds can be used to distinguish between vegetation types and show degradation patterns from litter into soil horizons. The study can have important impact and provide useful information (not only) for paleoenvironmental reconstructions. 
The only point that needs a bit more explanation in the current manuscript is how the endmember model which illustrates the degradation pattern (Figure 8) can be finally applied (e.g. in paleoenvironmental reconstruction) to correct for degradation effects as the authors state in their manuscript, but do not give a clear description how this can be actually done.

Some minor specific comments and suggestions are provided below and I hope they can still help to further improve the (already highly developed) manuscript.

II Specific comments

\section{Abstract}

At the end you mention that a "new n-alkane ratio is provided to illustrate, and tentatively account for, degradation effects of n-alkanes". It might be good to explicitly give this n-alkane ratio in the abstract, as it might be expected here by the reader.

\section{Introduction}

P2 L16 Average chain length (ACL) needs to be defined here at its first appearance.

P2 L31 Maybe you could describe here directly how you would "estimate the main contributing vegetation type to the soil based on n-alkyl leaf wax biomarker distribution". It is also not clear how your study would tackle (or even overcome) the potential pitfalls that you highlighted before.

\section{Material and Methods}

P3 L1 Change to "Altitude ranges from 16 to 899 m above sea level" (numbers should be from low to high).

P3 L6 Photographs are in Table S2. However, the descriptions of dominant plant species can be found in Table S1. Please change accordingly.

P3 L23 Isn't it important to stick to an exact timing for the derivatization, since the reac- 
tion might be incomplete if too short or derivatized compounds may become unstable if left too long at the high reaction temperatures? Therefore, the timing should also SOILD always be the same for all samples. I think "overnight" is not a good term to be used here. Maybe it was always $12 \mathrm{~h}$ or something similar, but it still seems quite long to me.

P3 L24 If you were not interested in the isotopes as well (GC-IRMS measurements), then these cleaning steps seem quite exhaustive. Were isotopes also measured on these samples?

Was there an internal standard used for the quantification of the alkanoic acids? If so please name it.

P3 L28 Does this include both odd and even homologues for both alkanes and alkanoic acids? Please clarify.

P3 L29 Some studies also normalize to the carbon content of soils instead of mass. Why did you choose to use normalization to mass and what difference could it make to the results?

P3 L33 Why is the C25 and the C35 not included in the ACL of alkanes?

P3 L34 Why is the C20, C22 and C34 not included in the ACL of alkanoic acids?

P3 L37/38 The OEP and EOP are mostly calculated with different formulas (that have two times the numerator and the denominator also two times but shifted by 2 carbon numbers, other authors also argue for shifting the nominator instead, see Freeman and Pancost in Treatise on Geochemistry 12, page 398). Why did you choose to use these "more simple" equations for OEP and EOP, and what difference could it make to the results?

P4 L1 Include "while" after the comma.

P4 L4-10 Is it not usual practice to first use the Levene Test to check for equal variances? If the assumption of equal variances is not fulfilled, then an ANOVA is not 
the appropriate test for significance, but a non-parametric test should be used (e.g. Kruskal-Wallis)? Also for the significance test between soil horizons I am not sure if ANOVA is the right method, since they are not independent of each other. Nevertheless, I am really not a friend of over-doing (and afterwards over-interpreting) statistics just for the sake of it. If the means with their standard errors are given (or boxplots like in this manuscript) everybody should be able to judge on their own if results are

Interactive different (and scientifically relevant, which I think is more important than p-values of inappropriate statistical tests).

\section{Results}

\section{P4 L35 Remove last bracket.}

P5 L1 What is the difference between OEP and CPI (apart from the name)?

P5 L20 Should it be "relative high n-C32 and n-C34"?

\section{Discussion}

P7 L14-32 Although Figure 8 is explained in detail, it still became not clear to me, how the "endmember plot" (Figure 8) can be finally applied to correct for degradation effects.

P8 L1-4 Again: How would you correct for degradation (e.g. by application within a paleoenvironmental study)?

\section{Conclusions}

P8 L20 How would you use the CDG indices quantitatively?

\section{References}

Sadly, the content of the book by A.P. Field is solely on statistics and does not include further sections on sex and drugs and rock 'n' roll :-)

For publications of the same authors, references should be sorted according to year (see for example those of Zech et al.). 
Other references not fully checked by reviewer. Please check again yourself that all references in the text are in the reference list and vice versa. Also check if all info is in SOILD the references.

\section{Figure 2}

Please also add the labels ( $L, A h 1, A h 2)$ in Figure $2 b$ to make it consistent with all the other figures. You could mention that the axes are in logarithmic scale.

\section{Figure 7}

Here the (a) and (c) are not visible. Please add to figure.

It is not clear (and also not described in the text) how the medians for vegetation types (d) are derived and what they mean. I guess, since there are only two points for grass and three for the other vegetation types, that each point represents the median of litter and soil horizons of the respective vegetation types. Please clarify.

What is the reason for using medians instead of means?

Interactive comment on SOIL Discuss., doi:10.5194/soil-2016-37, 2016. 